\title{
Comparison of 3-weekly cisplatin versus 3-weekly carboplatin in patients with locally advanced nasopharyngeal carcinoma receiving concurrent chemoradiotherapy: A multicentre analysis
}

\section{Arunee Dechaphunkul}

Prince of Songkla University Faculty of Medicine

\section{Pongwut Danchaivijitr}

Mahidol University Faculty of Medicine Siriraj Hospital

Rungarun Jiratrachu

Prince of Songkla University Faculty of Medicine

Tanadech Dechaphunkul

Prince of Songkla University Faculty of Medicine

Chatsuda Sookthon

Mahidol University Faculty of Medicine Ramathibodi Hospital

Chuleeporn Jiarpinitnun

Mahidol University Faculty of Medicine Ramathibodi Hospital

\section{Chudchanok Paoin}

Mahidol University Faculty of Medicine Siriraj Hospital

Jiraporn Setakornnukul

Mahidol University Faculty of Medicine Siriraj Hospital

\section{Bhoom Suktitipat}

Mahidol University Faculty of Medicine Siriraj Hospital

\section{Poompis Pattaranutaporn}

Mahidol University Faculty of Medicine Ramathibodi Hospital

Nuttapong Ngaimphaiboon ( $\sim$ Nuttapong.nga@mahidol.ac.th )

Mahidol University Faculty of Medicine Ramathibodi Hospital https://orcid.org/0000-0002-6535-6345

\section{Research article}

Keywords: nasopharyngeal carcinoma, chemoradiotherapy, cisplatin, carboplatin, nasopharyngeal carcinoma

Posted Date: December 30th, 2019 
DOI: https://doi.org/10.21203/rs.2.19749/v1

License: (c) (1) This work is licensed under a Creative Commons Attribution 4.0 International License. Read Full License 


\section{Abstract}

\section{Background}

Although concurrent chemoradiotherapy (CCRT) with high-dose cisplatin remains a standard of care for patients with locally advanced nasopharyngeal carcinoma (LA-NPC), carboplatin has alternatively been used, particularly for cisplatin-ineligible patients. However, the comparable efficacy of these two regimens remains unclear. The present study aimed to evaluate the efficacy and tolerability of 3-weekly carboplatin and 3-weekly cisplatin therapies.

\section{Methods}

From May 2005 to November 2014, we identified patients with LA-NPC treated with CCRT from a Thai multicentre head and neck cancer database. The patients were administered a chemotherapy (CT) regimen of either cisplatin $\left(75-100 \mathrm{mg} / \mathrm{m}^{2}\right.$ ) or carboplatin (AUC-5 or 6). Patient tolerability and survival were analysed and compared between regimens.

\section{Results}

Overall, 780 patients with LA-NPC were identified. Of these, the 76 patients $(9.7 \%)$ treated with carboplatin showed significantly more comorbidity and lower baseline creatinine clearance than those treated with cisplatin. Compared with the cisplatin group, a higher number of patients in the carboplatin group completed three planned cycles of CT during CCRT $(88.2 \%$ vs $52.0 \%, p<0.001)$ and three planned cycles of adjuvant CT ( $92.1 \%$ vs $78.7 \%, p=0.004)$. Furthermore, $28 \%$ of patients in the cisplatin group, as opposed to only $2.8 \%$ in the carboplatin group, required a dose reduction of CT in the subsequent cycle due to toxicities $(p<0.001)$. At the time of analysis, the 5 -year disease-free survival rate was $59.0 \%$ and $59.0 \%(p=0.935)$ and the 5 -year overall survival rate was $64.0 \%$ and $66.0 \%(p=0.530)$, in the cisplatin and carboplatin groups, respectively.

\section{Conclusions}

Carboplatin provided comparable efficacy to that of cisplatin but with better tolerability and could be considered as an alternative regimen, particularly in cisplatin-ineligible patients with LA-NPC. These findings still warrant a randomised phase III study to compare these two CT regimens.

\section{Background}

Nasopharyngeal carcinoma (NPC) is endemic to Southern China, the Middle East, Alaska, Greenland and Southeast Asia, including Thailand $(1,2)$. Concurrent chemoradiotherapy (CCRT) with high-dose cisplatin followed by three cycles of adjuvant chemotherapy (CT) containing cisplatin plus 5-fluorouracil is considered the standard protocol for patients with locally advanced nasopharyngeal carcinoma (LANPC), based upon the significant survival benefits demonstrated by the Intergroup (INT) 0099 study (3). The significant improvement of progression-free survival (PFS) and overall survival (OS) favouring 
combined modality over radiotherapy (RT) alone group was shown, with the 5-year PFS of $58 \%$ and 5year OS of $67 \%$ in the former group. Furthermore, a number of subsequent meta-analyses confirmed the added OS benefit of CCRT over RT alone (4-6). Nevertheless, the compliance rate of chemotherapy administration during CCRT in this protocol was quite low (63\%) owing to intolerable toxicities secondary to that of high-dose cisplatin (3). Because of this, alternative chemotherapy regimens have been used, particularly for cisplatin-ineligible patients.

Carboplatin is another platinum-based CT that has been shown to have similar radiosensitising properties but less oto-, renal- and gastrointestinal toxicities compared with cisplatin (7-9). Therefore, carboplatin has alternatively been used, particularly for cisplatin-ineligible patients. A number of studies have demonstrated the survival benefits of CCRT with carboplatin in patients with LA-NPC; however, the majority of the trials were phase II, single-arm studies $(10,11)$. Only one phase II randomised controlled trial directly compared weekly carboplatin therapy to standard 3-weekly cisplatin therapy and suggested that weekly carboplatin therapy had a non-inferior benefit with better tolerability in patients with LA-NPC (12). Conversely, a phase I/II study of CCRT with weekly carboplatin predicted that survival outcomes would be inferior to those of the experimental arm in the INT 0099 study (13). Taken together, the benefit of carboplatin use with CCRT in patients with LA-NPC needs to be better defined. Therefore, we aimed to evaluate the efficacies and tolerabilities of CCRT between 3-weekly carboplatin and 3-weekly cisplatin therapies in patients with LA-NPC in a multicentre study in Thailand.

\section{Methods}

From May 2005 to November 2014, we retrospectively reviewed medical information of newly-diagnosed NPC patients from a large multicentre, multidisciplinary database of Thai head and neck cancer patients encompassing three university hospitals in Thailand: Ramathibodi and Siriraj Hospitals, Mahidol University and Songklanagarind Hospital, Prince of Songkla University. The eligibility criteria for the study included patients with histologically-confirmed, non-metastatic NPC in stages II-IVb according to the $7^{\text {th }}$ edition of the American Joint Committee on Cancer Staging System (AJCC 2010) (14) and those who had received CCRT with either cisplatin $\left(75-100 \mathrm{mg} / \mathrm{m}^{2}\right.$ ) or carboplatin (AUC-5 or 6). A radical dose of RT was planned for all patients. Major exclusion criteria included patients with distant metastasis or previous radiation and/or chemotherapy, including induction CT for LA-NPC. The variables extracted from the database included patient information (date of birth, sex, vital status, date of death or date of last followup and cause of death), diagnosis (primary site and date of diagnosis), treatment (CT regimen, number of cycles of CT received, radiation technique and dose) and recurrence (date of recurrence and site of recurrence). Ethics approval was obtained through either the ethics committee or institutional review board at each study center and all patient information was de-identified.

\section{Statistical analysis}

Descriptive statistics were used to compare the data between the two groups of patients. OS was calculated from the date of diagnosis to the date of death from any causes. Disease-free survival (DFS) 
was calculated from the date of diagnosis to the first documented date of disease recurrence or date of death from any causes. Data for OS and DFS, respectively, were censored for patients who were still alive or free of recurrence at the last date of follow-up visit. Death status was validated and cross-checked with the Thai Social Security Death Index database. The last date of follow-up for censored patients was 11 June 2019. Survivals were calculated by using the Kaplan-Meier method. A log-rank test was used to compare the survival curves. A Cox proportional hazards model was used to estimate hazard ratios (HRs) and the corresponding $95 \%$ confidence intervals (Cls). A p-value of 0.05 or less was considered statistically significant. All analyses were performed using SPSS software, version 18.

\section{Results}

\section{Patients and baseline disease characteristics}

Between May 2005 and November 2014, we identified 780 eligible patients with newly-diagnosed LA-NPC who had received definitive CCRT with either 3-weekly cisplatin or 3-weekly carboplatin. Table 1 summarises the patient demographics and their baseline disease characteristics. A total of 76 of 780 patients $(9.7 \%)$ were treated with carboplatin during CCRT. Patients who received carboplatin were significantly associated with smoking $(p<0.001)$, higher incidences of comorbidity of vascular risk factors including hypertension, diabetes mellitus, dyslipidemia and/or previous vascular events $(p=0.014)$ or lower baseline creatinine clearance $(\mathrm{CCr})(p=0.001)$. The majority of patients in this cohort were diagnosed with stage IVa or IVb LA-NPC at the time of diagnosis $(43.4 \%$ and $48.7 \%$ in cisplatin and carboplatin groups, respectively).

\section{Treatment}

\section{Chemotherapy toxicity and tolerability}

More patients in the carboplatin group versus the cisplatin group completed three planned cycles of CT during CCRT $(88.2 \%$ vs $52.0 \%, p<0.001)$ and three planned cycles of adjuvant CT $(92.1 \%$ vs $78.7 \%$, $p=0.004$ ), as shown in Table 2 . Eighty-eight percent (624 out of 704) of patients in the cisplatin group and $95 \%$ (72 out of 76) in the carboplatin group were able to receive more than one cycle of CT during CCRT. Among these, $28.4 \%$ of patients in the cisplatin group, as opposed to only $2.8 \%$ in the carboplatin group, required a dose reduction of $\mathrm{CT}$ in the subsequent cycle due to toxicities $(p<0.001)$. However, there was no difference in the percentage of patients who required switching of CT regimen between the two groups $(p=0.566)$.

\section{Radiotherapy}

Intensity-modulated radiation therapy (IMRT) technique concurrently with CT as a definitive treatment was used significantly more in the cisplatin group (62.3\%) than in the carboplatin group $(0 \% ; p<0.001)$, as shown in Table 2. Almost all patients (74 out of 76) in the carboplatin arm received a 2D-technique. 
Nevertheless, the mean actual dose of RT was not significantly different between the two groups $(p=0.755)$.

\section{Survivals}

The median duration of follow-up was 6.2 years. The 5 -year OS of patients treated with cisplatin or carboplatin was $64.0 \%$ or $66.0 \%$, respectively ( $p=0.530$; HR $0.92,95 \% \mathrm{Cl}: 0.67-1.28$ ). However, at the time of analysis, 376 deaths had occurred, 335 in the cisplatin group and 41 in the carboplatin group. The median OS was 9.1 years in patients who had received cisplatin and 11.3 years in those who had received carboplatin, with no significant difference $(p=0.632)$ (Fig. 1).

The median DFS was 8.0 years in patients who had received cisplatin and 11.0 years in those who had received carboplatin, with no significant difference ( $p=0.882)$ (Fig. 2). The 5 -year DFS of patients treated with cisplatin or carboplatin was $59.0 \%$ or $59.0 \%$, respectively ( $p=0.935$; HR $0.98,95 \% \mathrm{Cl}: 0.71-1.34)$.

\section{Discussion}

In this study, we compared the clinical characteristics and survival outcomes for patients with LA-NPC who received definitive CCRT with either 3-weekly cisplatin or 3-weekly carboplatin. As expected, more patients in carboplatin group had comorbidity of vascular risk factors and lower baseline CCr; both factors are considered high risk for cisplatin ineligibility (15). In cisplatin-ineligible patients with locally advanced non-NPC head and neck squamous cell carcinoma, cetuximab is recommended for concurrent use with RT $(16,17)$. However, recommendation for the agent being used in cisplatin-ineligible patients with LA-NPC is limited. In standard guideline, carboplatin has been for decades recommended for concurrent use with RT (18), according to data from phase II studies (10-12). In addition, anti-EGFR monoclonal antibodies such as cetuximab and nimotuzumab were evaluated as radiosensitisers in patients with LA-NPC, albeit with limited data (19-23).

The INT 0099 study was the first landmark trial that demonstrated the survival benefits of CCRT with high-dose cisplatin followed by three cycles of adjuvant chemotherapy over RT alone in patients with LANPC; hence, this protocol has become standard practice (3). In our study, we observed comparable survival outcomes between patients who received cisplatin and those who received carboplatin. The 5year DFS and 5-year OS of patients treated with cisplatin in our study (59.0\% and $64.0 \%$, respectively) were similar to those reported by the INT 0099 study, which indicated a 5-year PFS of $58.0 \%$ and 5-year OS of $67.0 \%$. Interestingly, although more patients in our study were able to complete three cycles of adjuvant CT (78.7\%) in comparison to those in the INT 0099 study (55.0\%), the higher survival outcomes that we anticipated were not observed. A potential explanation might be due to the fact that the survival benefits of adjuvant CT after CCRT remain controversial $(24,25)$. In particular, the results of a phase III trial showed no significant improvements of survival when adjuvant CT was added to CCRT versus CCRT alone $(25,26)$. In terms of the carboplatin group, we found a slightly lower 3-year DFS $(66.0 \%)$ and 3-year OS (75.0\%) than those of the previous study (10), which reported a 3-year PFS of 72.7\% and 3-year OS of $89.7 \%$. This might be explained by the fact that there was a lower proportion of patients in our study 
$(88.2 \%)$ that were able to complete three planned cycles of carboplatin during CCRT as compared to the previous study (98.0\%). Hence, this finding might emphasise the importance of cumulative dose of CT.

One main issue for high-dose cisplatin is toxicity, leading to the low compliance rate. In our study, patients treated with carboplatin had a better compliance rate for CT than those treated with cisplatin. More patients in the carboplatin group than in the cisplatin group were able to complete planned cycles of CT. This result appeared similar to that of the INT 0099 study, which showed that only $63.0 \%$ and $55.0 \%$ of patients received three planned cycles of cisplatin during CCRT and adjuvant CT, respectively, and that the main reason for discontinuation of treatment was patient intolerance to toxicities (3). Additionally, we found significantly more patients in the cisplatin group who required a dose reduction for CT due to toxicities. In contrast, a study using 3-weekly carboplatin demonstrated that $98.0 \%$ of patients could complete planned cycles of CT (10). Taken together, this affirms a better tolerability of carboplatin over cisplatin in this setting.

IMRT is an advanced mode of RT that is expected to improve survival and reduce local toxicities; however, the superior survival benefits of IMRT over 2D-RT technique remains uncertain (27-32). Although the mean actual dose of RT was not different, the RT technique used was significantly dissimilar between the two arms of our study. IMRT was the most-used technique in the cisplatin group (62.3\%), whereas the 2DRT technique was practised the most in the carboplatin group (97.4\%). Though significantly fewer patients in cisplatin group were able to tolerate CT, we found no survival differences between the two arms. It is still hard to conclude whether these findings were affected by the enhanced benefit of IMRT. If the IMRT technique truly improves survival in patients with LA-NPC, the tolerability and survival outcome of patients receiving carboplatin concurrently with IMRT remains unknown because in our study, no patient in this arm received IMRT.

Our study is limited by its retrospective nature and relatively small number of patients in the carboplatin group. In addition, the selection bias of treating physicians is unavoidable in a retrospective study. However, given a larger sample size and longer duration of follow-ups, our study results were consistent with those of previous phase II studies that support the use of carboplatin CCRT in patients with LA-NPC .

\section{Conclusions}

Our study demonstrates that carboplatin provided comparable efficacy to that of cisplatin, but with better tolerability. Therefore, 3-weekly carboplatin concurrently with RT could be considered as a viable alternative regimen, particularly in cisplatin-ineligible patients with LA-NPC. Furthermore, carboplatin could serve as a better option in outpatient setting, given its short infusion without required fluid hydration. Carboplatin may perhaps become a preferred regimen at centres with limited access to IMRT. Importantly, our results still warrant a randomised phase III study to compare these two CT regimens, particularly in use with novel RT techniques such as IMRT.

\section{Abbreviations}


CCRT: Concurrent chemoradiotherapy

Cl: Confidence intervals

CT: Chemotherapy

DFS: Disease-free survival

HR: Hazard ratios

IMRT: Intensity-modulated radiation therapy

NPC: Nasopharyngeal carcinoma

OS: Overall survival

PFS: Progression-free survival

RT: Radiotherapy

RUN: Research University Network

\section{Declarations}

Ethics approval and consent to participate: Ethics approval was obtained through the ethics committee at the Ramathibodi (ID 07-59-48), and Siriraj (ECI 149/2562) hospitals, Mahidol university, and Songklanagarind hospital (REC.61-007-14-1), Prince of Songkla University, and all patient information was de-identified.

Consent for publication: Not applicable

Availability of data and materials: Not applicable

Competing interests: The authors declare that they have no competing interests.

Funding: The study was funded by the Thailand Grand Challenge Program for Research University Network (RUN) under the Precision Medicine for Cancer project by the National Research Council of Thailand (NRCT). The funding source has no involvement in the design of the study; data collection, analysis, and interpretation of data; and in writing the manuscript.

\section{Authors' contributions}

Study concepts: AD, NN

Study design: AD 
Data acquisition: PD, RJ, CS, CJ, CP, JS

Quality control of data and algorithms: BS, PP, NN

Data analysis and interpretation: $\mathrm{AD}, \mathrm{BH}, \mathrm{PP}, \mathrm{NN}$

Statistical analysis: $A D, T D$

Manuscript preparation: AD

Manuscript editing: RJ, CJ, JS, BS, PP, NN

Manuscript review: All authors

\section{Acknowledgements}

The study was funded by the Thailand Grand Challenge Program for Research University Network (RUN) under the Precision Medicine for Cancer project by the National Research Council of Thailand (NRCT). The authors thank Chanaphat Pundorlha, and Sirichai Srigate for assisting with data collection and verification.

\section{References}

1. Yu MC, Yuan JM. Epidemiology of nasopharyngeal carcinoma. Semin Cancer Biol. 2002;12(6):421-9.

2. Tangjaturonrasme N, Vatanasapt P, Bychkov A. Epidemiology of head and neck cancer in Thailand. Asia Pac J Clin Oncol. 2018;14(1):16-22.

3. Al-Sarraf M, LeBlanc M, Giri PG, Fu KK, Cooper J, Vuong T, et al. Chemoradiotherapy versus radiotherapy in patients with advanced nasopharyngeal cancer: phase III randomized Intergroup study 0099. J Clin Oncol. 1998;16(4):1310-7.

4. Baujat B, Audry H, Bourhis J, Chan AT, Onat H, Chua DT, et al. Chemotherapy in locally advanced nasopharyngeal carcinoma: an individual patient data meta-analysis of eight randomized trials and 1753 patients. Int J Radiat Oncol Biol Phys. 2006;64(1):47-56.

5. Blanchard P, Lee A, Marguet S, Leclercq J, Ng WT, Ma J, et al. Chemotherapy and radiotherapy in nasopharyngeal carcinoma: an update of the MAC-NPC meta-analysis. Lancet Oncol. 2015;16(6):645-55.

6. Zhang L, Zhao C, Ghimire B, Hong MH, Liu Q, Zhang Y, et al. The role of concurrent chemoradiotherapy in the treatment of locoregionally advanced nasopharyngeal carcinoma among endemic population: a meta-analysis of the phase III randomized trials. BMC Cancer. 2010;10:558.

7. Coughlin CT, Richmond RC. Biologic and clinical developments of cisplatin combined with radiation: concepts, utility, projections for new trials, and the emergence of carboplatin. Semin Oncol. 1989;16(4 Suppl 6):31-43. 
8. Douple EB, Richmond RC, O'Hara JA, Coughlin CT. Carboplatin as a potentiator of radiation therapy. Cancer Treat Rev. 1985;12 Suppl A:111-24.

9. Muggia FM. Overview of carboplatin: replacing, complementing, and extending the therapeutic horizons of cisplatin. Semin Oncol. 1989;16(2 Suppl 5):7-13.

10. Dechaphunkul T, Pruegsanusak K, Sangthawan D, Sunpaweravong P. Concurrent chemoradiotherapy with carboplatin followed by carboplatin and 5-fluorouracil in locally advanced nasopharyngeal carcinoma. Head Neck Oncol. 2011;3:30.

11. Songthong A, Chakkabat C, Kannarunimit D, Lertbutsayanukul C. Efficacy of intensity-modulated radiotherapy with concurrent carboplatin in nasopharyngeal carcinoma. Radiol Oncol. 2015;49(2):155-62.

12. Chitapanarux I, Lorvidhaya V, Kamnerdsupaphon P, Sumitsawan $Y$, Tharavichitkul E, Sukthomya V, et al. Chemoradiation comparing cisplatin versus carboplatin in locally advanced nasopharyngeal cancer: randomised, non-inferiority, open trial. Eur J Cancer. 2007;43(9):1399-406.

13. Parliament M, Jha N, Rapp E, Smith C, MacKinnon J, Nabholtz JM, et al. Concurrent weekly carboplatin and radiotherapy for nasopharyngeal carcinoma: report of a joint phase II study. Radiother Oncol. 2001;58(2):131-6.

14. Edge SB BD, Campton CC. AJCC cancer staging handbook from the AJCC cancer staging manual. $7^{\text {th }}$ ed. 38: Springer; 2011. p. 408.

15. Ahn MJ, D'Cruz A, Vermorken JB, Chen JP, Chitapanarux I, Dang HQ, et al. Clinical recommendations for defining platinum unsuitable head and neck cancer patient populations on chemoradiotherapy: $A$ literature review. Oral Oncol. 2016;53:10-6.

16. Bonner JA, Harari PM, Giralt J, Azarnia N, Shin DM, Cohen RB, et al. Radiotherapy plus cetuximab for squamous-cell carcinoma of the head and neck. N Engl J Med. 2006;354(6):567-78.

17. Mehanna H, Robinson M, Hartley A, Kong A, Foran B, Fulton-Lieuw T, et al. Radiotherapy plus cisplatin or cetuximab in low-risk human papillomavirus-positive oropharyngeal cancer (DeESCALaTE HPV): an open-label randomised controlled phase 3 trial. Lancet. 2019;393(10166):51-60.

18. Network NCC. Head and Neck Cancers (Version 3.2019) [Available from: https://www.nccn.org/professionals/physician_gls/pdf/head-and-neck.pdf.

19. Li Y, Chen QY, Tang LQ, Liu LT, Guo SS, Guo L, et al. Concurrent chemoradiotherapy with or without cetuximab for stage II to IVb nasopharyngeal carcinoma: a case-control study. BMC Cancer. 2017;17(1):567.

20. Wu X, Huang J, Liu L, Li H, Li P, Zhang J, et al. Cetuximab concurrent with IMRT versus cisplatin concurrent with IMRT in locally advanced nasopharyngeal carcinoma: A retrospective matched casecontrol study. Medicine (Baltimore). 2016;95(39):e4926.

21. Xu T, Liu Y, Dou S, Li F, Guan X, Zhu G. Weekly cetuximab concurrent with IMRT aggravated radiationinduced oral mucositis in locally advanced nasopharyngeal carcinoma: Results of a randomized phase II study. Oral Oncol. 2015;51(9):875-9. 
22. Wang F, Jiang C, Ye Z, Sun Q, Liu T, Xu M, et al. Efficacy and safety of nimotuzumab plus radiotherapy with or without cisplatin-based chemotherapy in an elderly patient subgroup (aged 60 and older) with nasopharyngeal carcinoma. Transl Oncol. 2018;11(2):338-45.

23. Kong L, Lin Q, Hu C, Xu T, Liao X, Shen C, et al. Radiation plus concurrent nimotuzumab versus cisplatin-based chemotherapy in locally advanced nasopharyngeal cancer: An interim analysis of a phase 3 randomized clinical trial. Elsevier; 2015. p. S129.24. Liang Z-G, Chen X-Q, Lin G-X, Yu B-B, Chen K-H, Zhong Q-L, et al. Significant survival benefit of adjuvant chemotherapy after concurrent chemoradiotherapy in locally advanced high-risk nasopharyngeal carcinoma. Sci Rep. 2017;7(1):41449.

24. Chen L, Hu CS, Chen XZ, Hu GQ, Cheng ZB, Sun Y, et al. Adjuvant chemotherapy in patients with locoregionally advanced nasopharyngeal carcinoma: Long-term results of a phase 3 multicentre randomised controlled trial. Eur J Cancer. 2017;75:150-8.

25. Chen L, Hu CS, Chen XZ, Hu GQ, Cheng ZB, Sun Y, et al. Concurrent chemoradiotherapy plus adjuvant chemotherapy versus concurrent chemoradiotherapy alone in patients with locoregionally advanced nasopharyngeal carcinoma: a phase 3 multicentre randomised controlled trial. Lancet Oncol. 2012;13(2):163-71.

26. Zhang B, Mo Z, Du W, Wang Y, Liu L, Wei Y. Intensity-modulated radiation therapy versus 2D-RT or 3DCRT for the treatment of nasopharyngeal carcinoma: A systematic review and meta-analysis. Oral Oncol. 2015;51(11):1041-6.

27. Marta GN, Silva V, de Andrade Carvalho H, de Arruda FF, Hanna SA, Gadia R, et al. Intensitymodulated radiation therapy for head and neck cancer: systematic review and meta-analysis. Radiother Oncol. 2014;110(1):9-15.

28. Co JL, Mejia MB, Que JC, Dizon JM. Effectiveness of honey on radiation-induced oral mucositis, time to mucositis, weight loss, and treatment interruptions among patients with head and neck malignancies: A meta-analysis and systematic review of literature. Head Neck. 2016;38(7):1119-28.

29. Zhang MX, Li J, Shen GP, Zou X, Xu JJ, Jiang R, et al. Intensity-modulated radiotherapy prolongs the survival of patients with nasopharyngeal carcinoma compared with conventional two-dimensional radiotherapy: A 10-year experience with a large cohort and long follow-up. Eur $\mathrm{J}$ Cancer. 2015;51(17):2587-95.

30. Peng G, Wang T, Yang KY, Zhang S, Zhang T, Li Q, et al. A prospective, randomized study comparing outcomes and toxicities of intensity-modulated radiotherapy vs. conventional two-dimensional radiotherapy for the treatment of nasopharyngeal carcinoma. Radiother Oncol. 2012;104(3):286-93.

31. OuYang PY, Shi D, Sun R, Zhu YJ, Xiao Y, Zhang LN, et al. Effect of intensity-modulated radiotherapy versus two-dimensional conventional radiotherapy alone in nasopharyngeal carcinoma. Oncotarget. 2016;7(22):33408-17.

\section{Tables}


Table 1: Patient demographics and baseline characteristics

Page $12 / 17$ 


\begin{tabular}{|c|c|c|c|c|}
\hline \multicolumn{2}{|c|}{ Characteristics } & \multicolumn{2}{|c|}{ Treatment group } & \multirow[t]{2}{*}{$p$-value } \\
\hline & & $\begin{array}{l}\text { Cisplatin } \\
\begin{array}{c}\mathrm{n}=704 \\
(\%)\end{array}\end{array}$ & $\begin{array}{c}\text { Carboplatin } \mathrm{n}=76 \\
(\%)\end{array}$ & \\
\hline \multirow[t]{3}{*}{ Median Age } & (years) & 61 & 58 & 0.903 \\
\hline & (Range) & $(16-77)$ & $(22-80)$ & \\
\hline & Age $\geq 65$ & $61(8.7)$ & $7(9.2)$ & 0.502 \\
\hline \multirow[t]{2}{*}{ Sex } & Male & $496(70.5)$ & $55(72.4)$ & 0.792 \\
\hline & Female & $208(29.5)$ & $21(27.6)$ & \\
\hline \multirow[t]{11}{*}{ ECOG PS } & 0 & $238(71.3)$ & $60(90.9)$ & 0.003 \\
\hline & 1 & $88(26.3)$ & $6(9.1)$ & \\
\hline & 2 & $8(2.4)$ & 0 & \\
\hline & Missing & 370 & 10 & \\
\hline & Smoker & $224(31.8)$ & $46(60.5)$ & $<0.001$ \\
\hline & Any Comorbidity & $93(13.2)$ & $18(23.7)$ & 0.014 \\
\hline & Hypertension & $72(10.2)$ & $13(17.1)$ & 0.057 \\
\hline & Diabetes & $32(4.5)$ & $4(5.3)$ & 0.473 \\
\hline & Hyperlipidaemia & $16(2.3)$ & 0 & 0.191 \\
\hline & Previous vascular & $8(1.1)$ & $1(1.3)$ & 0.605 \\
\hline & events & & & \\
\hline \multirow[t]{5}{*}{ T stage } & $\mathrm{T} 1$ & $153(21.8)$ & $11(14.5)$ & 0.389 \\
\hline & $\mathrm{T} 2$ & $213(30.4)$ & $24(31.6)$ & \\
\hline & T3 & $119(17.0)$ & $12(15.8)$ & \\
\hline & $\mathrm{T} 4$ & $216(30.8)$ & $29(38.1)$ & \\
\hline & Missing & 3 & 0 & \\
\hline \multirow[t]{4}{*}{$\mathrm{N}$ stage } & No & 77 (10.9) & $5(6.6)$ & 0.392 \\
\hline & N1 & $152(21.6)$ & $14(18.4)$ & \\
\hline & N2 & $358(50.9)$ & $46(60.5)$ & \\
\hline & N3 & $117(16.6)$ & $11(14.5)$ & \\
\hline
\end{tabular}




\begin{tabular}{|l|l|c|c|c|} 
Stage at & II & $109(15.5)$ & $11(14.5)$ & 0.681 \\
diagnosis & III & $289(41.1)$ & $28(36.8)$ & \\
& IVa-IVb & $506(43.4)$ & $37(48.7)$ & \\
\hline Histology & WHO type I & $204(55.1)$ & $38(50.0)$ & 0.381 \\
& WHO type II & $161(43.5)$ & $38(50.0)$ & \\
& WHO type III & 334 & 0 & 0.001 \\
& Missing & $91 \pm 26$ & $80 \pm 25$ & \\
\hline & Mean baseline CCr* \pm & & \\
& SD & & & \\
& $\left(\mathrm{mL} / \mathrm{min} / 1.73 \mathrm{~m}^{2}\right)$ & & & \\
\hline
\end{tabular}

*CCr-creatinine clearance calculated by Cockcroft-Gault formula

Table 2: Treatment modality and tolerability of patients with LA-NPC treated with carboplatin or cisplatin CCRT 


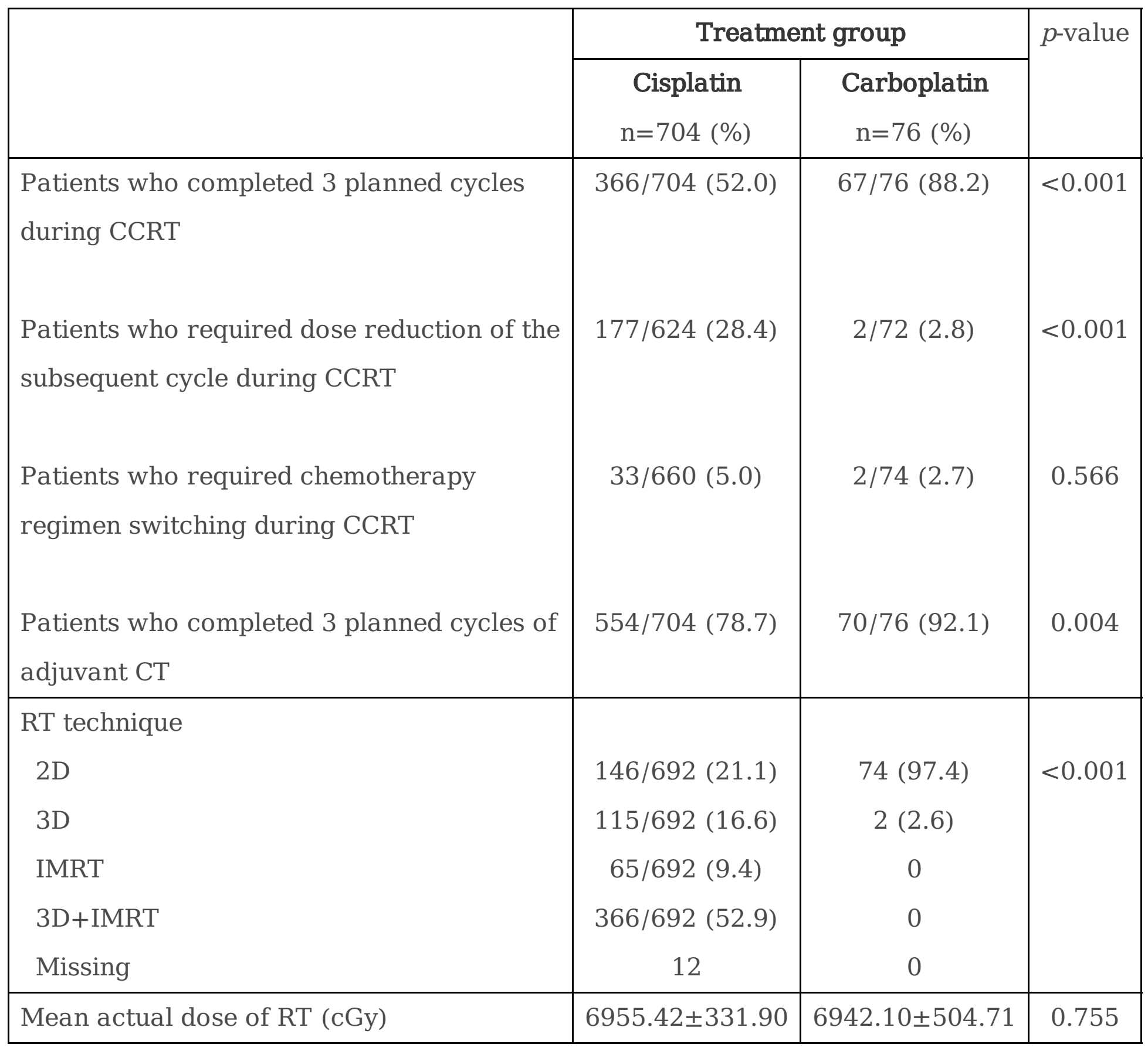

\section{Figures}




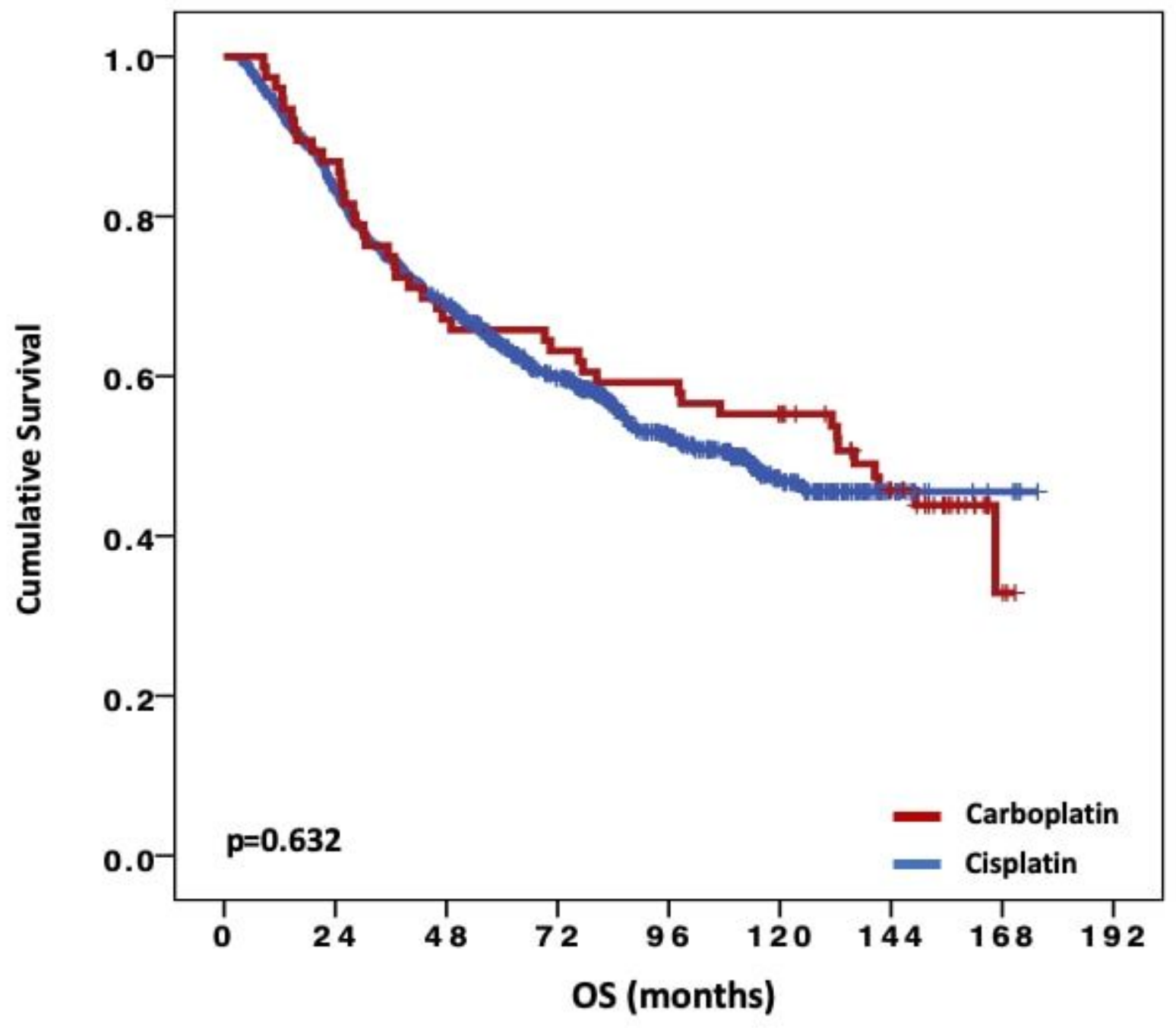

Figure 1

Overall survival (OS) of patients with LA-NPC treated with carboplatin or cisplatin CCRT 


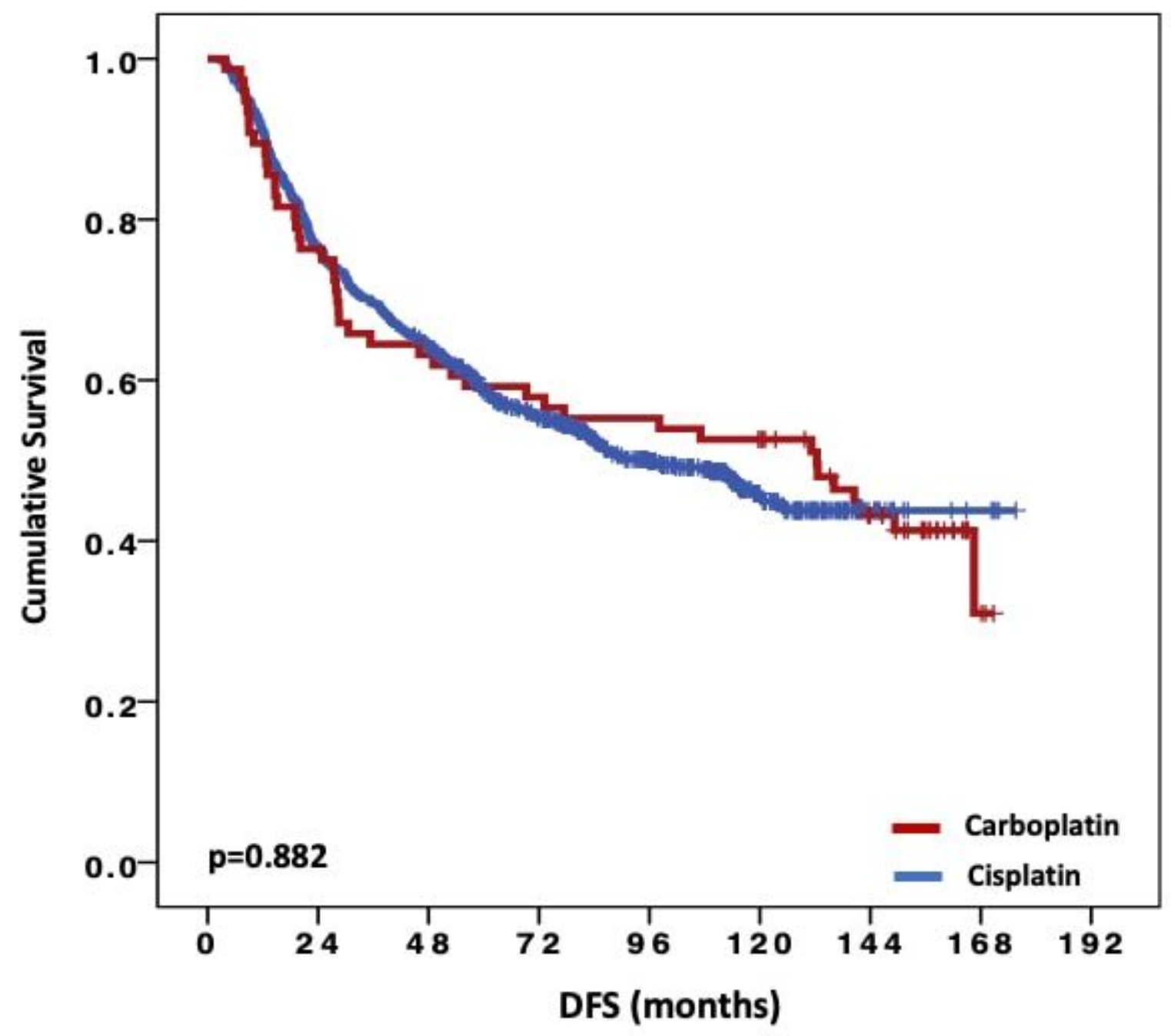

Figure 2

Disease-free survival (DFS) of patients with LA-NPC treated with carboplatin or cisplatin CCRT 